\title{
Predictors of neurologists confirming or overturning emergency physicians' diagnosis of TIA or stroke
}

\author{
Miguel A. Cortel-LeBlanc ${ }^{1} \cdot$ Mukul Sharma $^{14} \cdot$ Achelle Cortel-LeBlanc $^{3} \cdot$ Marco L. A. Sivilotti $^{4} \cdot$ Marcel Émond $^{5,6}$. \\ Ian G. Stiell ${ }^{1,2} \cdot$ Grant Stotts $^{7}$. Jacques Lee ${ }^{8,9} \cdot$ Andrew Worster $^{10} \cdot$ Judy Morris $^{11} \cdot$ Ka Wai Cheung $^{12}$. \\ Albert Y. Jin ${ }^{13}$. Wieslaw J. Oczkowski ${ }^{14}$. Demetrios J. Sahlas ${ }^{14} \cdot$ Heather E. Murray ${ }^{4}$. Ariane Mackey ${ }^{5,15}$. \\ Steve Verreault ${ }^{5,15} \cdot$ Marie-Christine Camden $^{5,15}$. Samuel Yip ${ }^{16} \cdot$ Philip Teal $^{16} \cdot$ David J. Gladstone $^{17}$. \\ Mark I. Boulos ${ }^{17}$. Nicolas Chagnon ${ }^{18}$. Elizabeth Shouldice ${ }^{3}$. Clare Atzema ${ }^{9} \cdot$ Tarik Slaoui $^{11}$. Jeanne Teitelbaum ${ }^{11}$. \\ Kasim E. Abdulaziz ${ }^{2} \cdot$ George A. Wells $^{2} \cdot$ Monica Taljaard $^{2} \cdot$ Jeffrey J. Perry ${ }^{1,2}$ (1)
}

Received: 19 March 2021 / Accepted: 19 July 2021 / Published online: 1 September 2021

(C) The Author(s), under exclusive licence to Canadian Association of Emergency Physicians (CAEP)/ Association Canadienne de Médecine d'Urgence (ACMU) 2021

\begin{abstract}
Background Transient ischemic attack (TIA) and non-disabling stroke are common emergency department (ED) presentations. Currently, there are no prospective multicenter studies determining predictors of neurologists confirming a diagnosis of cerebral ischemia in patients discharged with a diagnosis of TIA or stroke. The objectives were to (1) calculate the concordance between emergency physicians and neurologists for the outcome of diagnosing TIA or stroke, and (2) identify characteristics associated with neurologists diagnosing a stroke mimic.

Methods This was a planned sub-study of a prospective cohort study at 14 Canadian EDs enrolling patients diagnosed with TIA or non-disabling stroke from 2006 to 2017. Logistic regression was used to identify factors associated with neurologists' diagnosis of cerebral ischemia. Our primary outcome was the composite outcome of cerebral ischemia (TIA or non-disabling stroke) based on the neurologists' assessment.

Results The diagnosis of cerebral ischemia was confirmed by neurologists in 5794 patients (55.4\%). The most common identified stroke mimics were migraine (18\%), peripheral vertigo (7\%), syncope (4\%), and seizure (3\%). Over a third of patients (38.4\%) ultimately had an undetermined aetiology for their symptoms. The strongest predictors of cerebral ischemia confirmation were infarct on CT (OR 1.83, 95\% CI 1.65-2.02), advanced age (OR comparing 75th-25th percentiles 1.67, 1.55-1.80), language disturbance (OR 1.92, 1.75-2.10), and smoking (OR 1.67, 1.46-1.91). The strongest predictors of stroke mimics were syncope (OR 0.59, 0.48-0.72), vertigo (OR 0.52, 0.45-0.59), bilateral symptoms (OR 0.60, 0.50-0.72), and confusion (OR 0.50, 0.44-0.57).

Conclusion Physicians should have a high index of suspicion of cerebral ischemia in patients with advanced age, smoking history, language disturbance, or infarcts on CT. Physicians should discriminate in which patients to pursue stroke investigations on when deemed at minimal risk of cerebral ischemia, including those with isolated vertigo, syncope, or bilateral symptoms.
\end{abstract}

Keywords TIA $\cdot$ Stroke $\cdot$ Stroke mimic $\cdot$ Cerebral ischemia $\cdot$ Emergency department

Previous presentations: The work described in this study was presented at the Canadian Association of Emergency Physicians (CAEP) Annual Conference (May 2018) in Calgary, Alberta, and at the Society for Academic Emergency Medicine (SAEM) annual meeting in Indianapolis, Indiana (May 2018).

Jeffrey J. Perry

jperry@ohri.ca

Extended author information available on the last page of the article 


\section{Résumé}

Contexte L'accident ischémique transitoire (AIT) et l'accident vasculaire cérébral (AVC) non invalidant sont des présentations courantes dans les services d'urgence. Actuellement, il n'existe pas d'études prospectives multicentriques déterminant les facteurs prédictifs de la confirmation par les neurologues d'un diagnostic d'ischémie cérébrale chez les patients sortis de l'hôpital avec un diagnostic d'AIT ou d'AVC. Les objectifs étaient de (1) calculer la concordance entre les urgentistes et les neurologues pour le résultat du diagnostic de l'AIT ou de l'AVC, et (2) identifier les caractéristiques associées au diagnostic par les neurologues d'une imitation d'AVC.

Méthodes Il s'agissait d'une sous-étude planifiée d'une étude de cohorte prospective dans 14 services d'urgence canadiens recrutant des patients diagnostiqués avec un AIT ou un AVC non invalidant de 2006 à 2017. Une régression logistique a été utilisée pour identifier les facteurs associés au diagnostic d'ischémie cérébrale par les neurologues. Notre résultat principal était le résultat composite de l'ischémie cérébrale (AIT ou accident vasculaire cérébral non invalidant) selon l'évaluation des neurologues.

Résultats Le diagnostic d'ischémie cérébrale a été confirmé par des neurologues chez 5794 patients $(55,4 \%)$. Les imitateurs d'AVC identifiés les plus courants étaient la migraine (18\%), le vertige périphérique (7\%), la syncope (4\%) et les convulsions (3\%). Plus d'un tiers des patients $(38,4 \%)$ avaient finalement une étiologie indéterminée pour leurs symptômes. Les prédicteurs les plus forts de la confirmation de l'ischémie cérébrale étaient l'infarctus au scanner (OR 1.83, IC $95 \%$ 1.65-2.02), l'âge avancé (OR comparant les 75e et 25e percentiles 1.67, 1.55-1.80), les troubles du langage (OR 1.92, 1.75-2.10) et le tabagisme (OR 1.67, 1.46-1.91). Les prédicteurs les plus forts d'imitateurs d'AVC étaient la syncope (OR 0.59, 0.48-0.72), le vertige (OR 0.52, 0.45-0.59), les symptômes bilatéraux (OR 0.60, 0.50-0.72) et la confusion (OR 0.50, 0.44-0.57).

Conclusion Les médecins devraient avoir un indice élevé de suspicion d'ischémie cérébrale chez les patients ayant un âge avancé, des antécédents de tabagisme, des troubles du langage ou des infarctus au scanner. Les médecins doivent distinguer les patients sur lesquels poursuivre des investigations sur un AVC lorsqu'ils sont jugés à risque minimal d'ischémie cérébrale, y compris ceux présentant des vertiges isolés, une syncope ou des symptômes bilatéraux.

\section{Clinician's capsule}

\section{What is known about the topic?}

Early initiation of stroke prevention treatment in patients with TIA can reduce stroke recurrence.

\section{What did this study ask?}

We examined the rate of neurologists confirming cerebral ischemia in patients diagnosed with TIA or minor stroke by emergency physicians.

\section{What did this study find?}

Cerebral ischemia was confirmed in $55.4 \%$ of patients, and we identified characteristics associated with confirmation of cerebral ischemia.

\section{Why does this study matter to clinicians?}

Improved accuracy in diagnosing TIA may avoid unnecessary investigations and treatments, and early initiation of stroke prevention treatments when indicated.

\section{Introduction}

Better prevented than treated, strokes are often catastrophic events [1,2]. Transient ischemic attacks (TIAs), and the closely related mild, non-disabling strokes, offer a unique but highly time-sensitive opportunity for stroke prevention, with a 90 -day subsequent major stroke risk of $10 \%$ or higher overall, and over 50\% in some in the absence of treatment [3-7]. Yet the diagnosis of a TIA is challenging as it is based on patient history given the typically rapid resolution of the cardinal neurologic symptoms prior to physician assessment. Thus, emergency physicians are often asked to distinguish between those patients whose transient neurologic symptoms represent bona fide cerebral ischemia and are, therefore, likely to benefit from rapid advanced investigations and urgent referral to a neurologist, and those patients more likely to have experienced a stroke or TIA mimic.

While emergency physicians do not wish to miss a TIA, overdiagnosis in the emergency department contributes to increased cost, exposure to unnecessary tests, increased harm from unwarranted anti-thrombotic medications and radiation as well as delayed care for bona fide TIA patients [8-12]. The Canadian Stroke Best Practice Recommendations advise that patients presenting with suspected TIA should be evaluated with laboratory investigations, 12-lead ECG, brain imaging (CT or MRI), and vascular imaging preferably with CTA or MRI [13]. While thorough patient assessment and early stroke prevention therapy has decreased the risk for subsequent stroke [7], the cost, harms and prioritization of TIA work-up mandates careful patient selection. Previous studies report that consultant neurologists confirm the referring physicians' suspicion of TIA in as few as $22 \%$ to as many as $90 \%$ of cases [12, 14-17]. This wide range reflects different settings, but also suggests a wide range of overdiagnosis, inefficient care, and unnecessary investigations, referral and treatment. 
The purpose of this study was (1) to calculate the rate of concordance between emergency physicians' working diagnosis of possible TIA or stroke and neurologists' final diagnostic impression for the cerebral ischemia (i.e. TIA or non-disabling stroke) and (2) to identify patient characteristics associated with neurologists confirming the referring diagnosis of possible TIA or stroke, or deeming the original presentation to have represented a stroke or TIA mimic.

\section{Methods}

We had previously conducted a prospective cohort study at 14 Canadian emergency departments (ED) assessing clinical characteristics of adult patients diagnosed with possible TIA or non-disabling stroke from 2006 to 2017 [18, 19]. In this planned sub-study, we assessed the cohort who were evaluated by a neurologist either immediately or within 90 days.

Patients were excluded if they were diagnosed with a disabling stroke (i.e. could not manage activities of daily living) in the ED, had an altered level of consciousness [i.e. Glasgow Coma Scale (GCS) score < 15], presented more than 7 days after symptom onset, received thrombolytics or intravascular thrombectomy, or if they were not assessed by a neurologist. This study was approved by each institution's Research Ethics Board (REB) and the need for explicit written consent from patients was waived in this minimal risk observational study. Verbal informed consent was obtained for telephone follow-ups.

All patients were initially assessed by emergency physicians who completed standardized data collection forms at the time of the index ED visit that included history, physical examination, past medical history, and investigations. Data collection forms were completed without knowledge of the neurologists' final diagnoses. All patients enrolled in the study were referred to an outpatient stroke prevention clinic staffed by neurologists. Information about patients' follow-up visits with neurology was obtained by trained research personnel performing structured, 90-day medical record review of hospital, ED and outpatient clinic visits, and of diagnostic imaging. Patients were also contacted by telephone at 7 and 90 days to identify any subsequent stroke or neurologic symptoms. Every possible subsequent stroke was adjudicated by a local committee of three physicians with expertise in stroke.

Our primary outcome was the composite outcome of cerebral ischemia, defined as TIA or non-disabling ischemic stroke at the time of the index visit. Our reference standard for final diagnosis was based on all notes by the consultant neurologist, whether generated during hospital admission or outpatient clinic follow-up, as extracted by study research personnel. These personnel extracted the neurologists' most probable diagnoses for the index event as based on their final assessment within 90 days of the initial emergency department visit. This final diagnosis was further classified as being "cerebral ischemia" (i.e. TIA or stroke) if any qualifying language included the terms "possible" or "may have" but not "unlikely" or "do not believe", "not cerebral ischemia" if a different diagnosis was deemed more likely (i.e. stroke or TIA mimic), and "unknown" when the final diagnosis included language such as "not yet determined (NYD)".

We calculated descriptive statistics and used logistic regression to identify factors associated with a final diagnosis of cerebral ischemia using nineteen pre-selected candidate variables based on clinical judgment (Supplemental, eTable 1). Multiple $(N=10)$ imputation was used to deal with missing values on predictor variables. The imputation model included all candidate variables as well as the dependent variables, and imputations were generated using aregImpute function (Hmisc package). Variance inflation factors (VIFs) were used to examine potential multicollinearity prior to analysis. The continuous variable "age" was modelled using a restricted cubic spline function with 3 knots at the 10th, 50th, and 90th percentiles. The association between each predictor and the outcome was expressed using odds ratios (OR) and 95\% confidence intervals (CI). For the continuous variable "age", the ORs were expressed comparing 75th-25th percentile values. A more parsimonious approximation to the full model was derived using the fast step-down procedure within the rms package of $\mathrm{R}$ [20]. To assess the performance of the model, we used 1,000 bootstrap samples, with the step-down procedure applied in every step, and calculated the optimism-corrected C-statistic [21]. Statistical significance was assessed at the 0.05 level. All analyses were conducted using R programming language build 3.4 and SAS 9.4 statistical software.

\section{Data availability}

Requests for sharing of the de-identified data will be considered and reviewed by the study's steering committee. Requests can be made to the corresponding author.

\section{Results}

Overall, 11,508 patients were referred to a consultant neurologist by emergency physicians for possible TIA or nondisabling stroke and enrolled prospectively into the study; another 2927 patients were potentially eligible based on medical record review but did not have a study form completed by the treating physician and were thus deemed missed (Fig. 1). Of the patients referred, 1042 patients were 


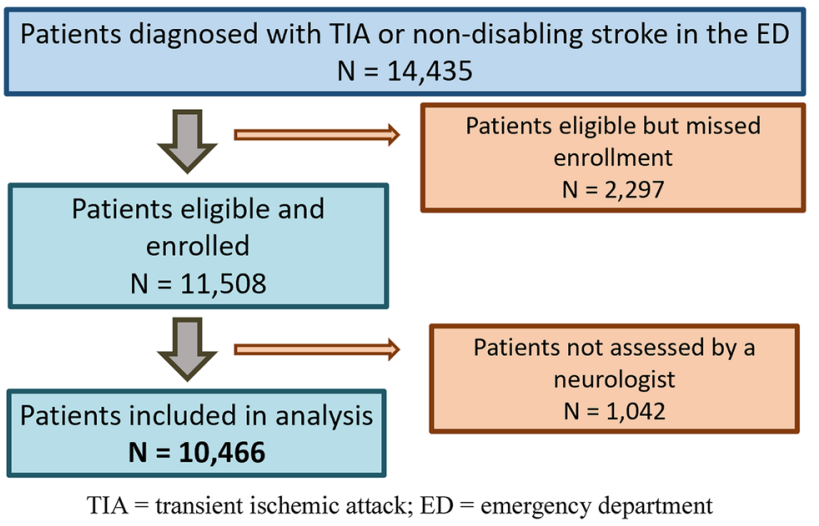

Fig. 1 Study flow for patients presenting with TIA or non-disabling stroke to the emergency department

not assessed by a neurologist, and thus removed. In total, 10,466 patients were included in our analysis. Overall, $20.5 \%$ of patients had missing data on at least one candidate predictive variable.

The mean patient age was 68.1 years ( 75 th percentile of 80 years), and just over half were female (Table 1). Most patients presented with their first ever TIA or non-disabling stroke (72.5\%). Computed tomography (CT) and magnetic resonance imaging (MRI) were performed on $97.0 \%$ and $12.7 \%$ of patients, respectively. Cerebral infarcts were identified in $28.0 \%$ of CT and in $46.2 \%$ of MRI studies obtained. A minority of patients $(7.2 \%)$ were admitted on their index visit and $18.3 \%$ of patients were assessed by a neurologist in the ED.

Table 1 Baseline characteristics of patients diagnosed with TIA and non-disabling stroke in the emergency department

\begin{tabular}{ll}
\hline Characteristics & Patients $(N=10,466)$ \\
\hline Demographics (\%) & $68.1(14.5)$ \\
Mean age in years (SD) & $5397(51.6)$ \\
Female, $\%$ & $7555(72.5)$ \\
First ever TIA or stroke, $(N=10,419)$ & \\
Baseline medications (\%) & $4187(40.0)$ \\
Antiplatelets & $540(5.2)$ \\
Warfarin & $339(3.2)$ \\
Other anticoagulants & \\
Past medical history (\%) & $1788(17.1)$ \\
Coronary artery disease & $6173(59.0)$ \\
Hypertension & $1030(9.8)$ \\
Atrial fibrillation & $1969(18.8)$ \\
Diabetes & $1229(11.7)$ \\
Smoker & \\
Outcomes (\%) & $5794(55.4)$ \\
Cerebral ischemia confirmed by a neurologist &
\end{tabular}

$S D$ standard deviation, TIA transient ischemic attack
Table 2 Neurologists' final diagnosis of patients initially diagnosed with TIA and non-disabling stroke in the emergency department

\begin{tabular}{lc}
\hline Diagnosis & Patients $(N=10,466)$ \\
\hline Confirmation of cerebral ischemia-TIA or & $5794(55.4)$ \\
Stroke (\%) & \\
Migraines and headaches & $825(17.7)$ \\
Peripheral vertigo and dizziness & $333(7.1)$ \\
Syncope and pre-syncope & $191(4.1)$ \\
Seizure & $157(3.4)$ \\
TGA & $116(2.5)$ \\
Bell's palsy & $63(1.4)$ \\
Anxiety & $53(1.1)$ \\
Symptoms NYD & $1792(38.4)$ \\
Other non-neurological disorders & $834(17.9)$ \\
Other neurological diagnoses & $308(6.6)$ \\
\hline
\end{tabular}

TIA transient ischemic attack, TGA transient global amnesia, $N Y D$ not yet determined

Neurologists agreed with the final diagnosis of cerebral ischemia in 5,794 patients (55.4\%) (Table 2). The most common identified cerebral ischemia mimics were migraine (18\%), peripheral vertigo (7\%), syncope (4\%), and seizure (3\%). In over a third of patients (38.4\%) whom neurologists felt had a diagnosis other than TIA or stroke, the final diagnosis of "symptoms not yet determined" or of unknown aetiology was made. The rate of subsequent stroke within 90 days in those with confirmation of cerebral ischemia was $4.7 \%$ compared to $0.5 \%$ when neurologists overturned the diagnosis of TIA or stroke. In patients for whom cerebral ischemia was not confirmed, the rate of subsequent stroke within 90 days was $0.2 \%$ when neurologists determined a specific alternate diagnosis and $0.8 \%$ when the final diagnosis was "symptoms not yet determined".

Table 3 presents the results from the logistic regression analysis after applying the step-down procedure, identifying features associated with cerebral ischemia confirmation as compared to a mimic. No substantial multicollinearity was detected. The strongest predictors of cerebral ischemia confirmation were infarct on CT (OR 1.83, 95\% CI 1.65-2.02), advanced age (OR comparing 75th-25th percentiles 1.67, 1.55-1.80), language disturbance (OR 1.92, 1.75-2.10), and smoking (OR 1.67, 1.46-1.91). Conversely, the strongest predictors of stroke mimics were syncope (OR 0.59, 0.48-0.72), vertigo (OR 0.52, 0.45-0.59), bilateral symptoms (OR 0.60, $0.50-0.72$ ), and confusion (OR 0.50, 0.44-0.57). The optimism-corrected C-statistic was 0.70 (CI 0.69-0.71).

\section{Discussion}

In our study of 10,466 patients diagnosed with TIA or nondisabling stroke in the ED, we found the rate of confirmation for the diagnosis of TIA or non-disabling stroke 
Table 3 Results from logistic regression analysis of variables associated with cerebral ischemia confirmation after applying the stepdown procedure

\begin{tabular}{ll}
\hline Variable & Odds ratio (95\% CI) \\
\hline Demographics & \\
Age (75th vs. 25th percentile) & $1.67(1.55-1.80)$ \\
Female & $0.71(0.65-0.77)$ \\
History or exam & \\
Vertigo & $0.52(0.45-0.59)$ \\
Confusion & $0.50(0.44-0.57)$ \\
Syncope & $0.59(0.48-0.72)$ \\
Language disturbance & $1.92(1.75-2.10)$ \\
Gait disturbance & $1.36(1.22-1.51)$ \\
Abnormal physical exam & $1.25(1.15-1.36)$ \\
Bilateral symptoms & $0.60(0.50-0.72)$ \\
Past medical history & \\
Smoking & $1.67(1.46-1.91)$ \\
Investigations & \\
Infarct on CT & $1.83(1.65-2.02)$ \\
\hline
\end{tabular}

TIA transient ischemic attack, CT computed tomography, CIconfidence interval, Age 75th percentile $=80$ years, 25 th percentile $=58$ years

between emergency physicians and neurologists to be $55.4 \%$. Migraines were the most common mimicker of cerebral ischemia (18\%) consistent with previously reported studies, followed by vertigo (7\%), and syncope (4\%) [12]. The incidence of seizures (3\%) as a stroke or TIA mimic in our study was lower than in a systematic review from various settings in which seizures were diagnosed in $19.6 \%$ of patients with suspected stroke [22]. We found six variables to be modestly predictive of the neurologist confirming cerebral ischemia: increasing age, language disturbance, gait disturbance, abnormal physical exam, smoking, and presence of infarct on CT. Conversely, female sex, vertigo, confusion, syncope, and bilateral symptoms all predicted an alternate diagnosis. The risk of subsequent major stroke within 90 days was $4.7 \%$ in patients with cerebral ischemia confirmation, compared to $0.5 \%$ in patients with an alternate diagnosis.

The $55.4 \%$ cerebral ischemia-diagnosis confirmation rate found in our study is lower than three previously reported studies; however, significant differences distinguish our study. Kothari conducted a review of 446 patients admitted with stroke and found a diagnosis confirmation rate of 98.6\%; however, our population included TIA and nondisabling stroke patients who were mostly discharged rather than the sicker cohort in their study [16]. It is not unexpected that in a group of patients with prominent, persistent deficits that the concordance is much higher. Moreover, the prospective aspect of our study reduces the risk of selection bias that retrospective studies introduce. Morgenstern analysed
1,800 TIA or stroke patients in the ED from a prospectively enrolled surveillance database and found a confirmation rate of $89.1 \%$ [17]. Two notable differences in their study from ours include their significantly higher admission rate (82\%), and their determination of TIA confirmation from neurologists reviewing hospital documentation and results without examining the patient. Lastly, Merino analysed prospectively collected data of 8,187 patients who were assessed by a stroke team and found a confirmation rate of 70\% [23]. A major difference between their study and ours is that they analysed patients referred to the stroke team for assessment in the $\mathrm{ED}(75 \%)$, or as inpatients (25\%), while our study focused on outpatient referrals for TIA and non-disabling stroke.

In contrast to the aforementioned studies, our cerebral ischemia confirmation rate of $55.4 \%$ is significantly higher than that found by Lee in 2015 of $22 \%$ [12]. Their study included referrals from general practitioners and other hospital units in addition to emergency physician referrals. Furthermore, their study is limited by a small sample size of 82 patients.

Our finding that female sex was identified as an independent predictor of an alternate diagnosis must be interpreted carefully. Previously, female sex had also been associated with higher likelihood of a diagnosis of stroke mimic [23, 24]. Moreover, rates of misclassification of stroke mimic and cerebral ischemia are higher in women than in men [25] and the prevalence of atypical presentations of stroke is higher in women [26, 27]. These findings could potentially be partially explained by the higher prevalence of migraine in women [28], which can both manifest as a true stroke mimic but also increase the likelihood of stroke [29-31]. Thus, given the existing tendency to underestimate the likelihood of TIA or stroke in women [32], one must be cautious to not overturn a suspicion of cerebral ischemia based on the patient's sex.

In our study, vertigo predicted an alternate diagnosis other than cerebral ischemia. Dizziness and vertigo are common presenting complaints in the ED, and can potentially be due to posterior circulation cerebral ischemia [33, 34]. While the prevalence of cerebrovascular etiologies explaining vertigo is low compared to vestibular and cardiovascular causes [33], clinicians should ensure no other findings of posterior circulation cerebral ischemia (e.g. pathologic nystagmus, dysmetria, ataxia, diplopia, dysarthria, and lateralizing weakness to name a few) are present in patients with vertigo before making the diagnosis of a stroke mimic.

A strength of our study is that it is a large multicenter prospective cohort study involving 14 different EDs. All patients were seen and enrolled in the ED, and we extracted a large number of historical, clinical, and diagnostic variables. Further, 90-day stroke outcomes were adjudicated by blinded committees of stroke specialists including two stroke neurologists and 
one experienced emergency physician (two sites without two stroke neurologists used two experienced emergency physicians and if they disagreed with the local stroke neurologist, an external stroke neurologist verified their adjudication).

\section{Limitations}

Our study has some limitations. First, the transition from a "time-based" to a "tissue-based" definition of TIA presents challenges due to the variable use of MRI. In an attempt to mitigate this issue, we used a combination of "TIA" and "stroke" to determine cerebral ischemia confirmation to capture all focal cerebral ischemic events. While we believe that this is a small limitation as the goal of TIA and stroke care is the prevention of a subsequent stroke, MRI has been shown to potentially confirm cerebral ischemia in patients who were deemed to clinically not have experienced a TIA or stroke and will likely have an increasingly important role in cases of suspected TIA with negative CT [25].

Second, approximately $20 \%$ of patients had missing values on at least one predictor variable; however, no patients were excluded from analysis as we used state of the art multiple imputation methodology for dealing with missing values. No single variable had more than 5\% missing values. Given that the multiple imputation procedure used all available information to create a complete dataset for analysis, we do not anticipate substantial bias due to missing values.

Third, our gold standard diagnosis for cerebral ischemia was based on a neurologist's clinical evaluation of the patient and interpretation of all the tests and information available. Since the timing of neurology consultation was often after the patient's index ED visit (i.e. within 90 days), neurologists would have more information available (e.g. CT, MRI, carotid Doppler, echocardiograms) to help them in making the diagnosis. MRI was only performed on $12.7 \%$ of patients; however, this reflects the practice of most Canadian tertiary stroke centres.

Fourth, some emergency physicians may not have definitively diagnosed a TIA or minor stroke but referred patients to a neurologist for further assessment to "rule out" this diagnosis. As such there may be some misclassification in both directions: falsely concordant when emergency physicians did not really think that it was cerebral ischemia but with further time and assessments this diagnosis was confirmed, versus falsely discordant when emergency physicians thought that it was cerebral ischemia but new information available after the index visit allowed neurologists to rule out this diagnosis. Although the degree of misclassification is difficult to quantify, we believe that its impact in our study was relatively small given that most patients had most of their testing (including cerebral imaging) completed in the ED.

Fifth, our study did not delineate anterior circulation vs. posterior circulation stroke syndromes. The presentation of posterior circulation cerebral ischemia is unique and mandates a comprehensive cerebellar and brainstem examination. Further studies would be needed to explore whether the predictors of cerebral ischemia differ between anterior and posterior circulation events.

Lastly, it is also possible that our study underestimates the confirmation rate, given that neurologists had a final diagnosis of "symptoms not yet determined" in over $1 / 3$ of patients. At least some of these patients likely had a true cerebral ischemic event at the time of their initial assessment. In a recent prospective study of patients with low-risk TIA symptoms, MRI identified infarcts in $13.5 \%$ of patients, the diagnosis was altered in $30 \%$ of patients, and the risk of recurrent stroke at 1 year was $0.7 \%$, similar to the $0.5 \%$ risk of recurrent stroke within 90 days in the patients in our cohort for whom neurologists disagreed with the diagnosis of cerebral ischemia [35]. Of the patients that neurologists made an identifiable diagnosis (i.e., excluding those with final diagnosis of "symptoms NYD"), the diagnosis of TIA or non-disabling stroke was confirmed in $66.8 \%$ of patients. Since our cohort represents only patients referred from the $\mathrm{ED}$, it is possible that the incidence of stroke mimics in other settings would differ.

\section{Future research}

Future studies should explore the safety of using a set of clinical predictors to rule out cerebral ischemia in patients deemed at low risk of TIA or stroke, including patient outcomes and resource utilization. Moreover, how the prognosis of patients with clinical predictors for stroke-mimics identified in this study compares between patients with and without cerebral ischemia on MRI remains to be elucidated.

\section{Conclusions}

We have identified a set of patient characteristics that are associated with confirmation of cerebral ischemia by neurologists and presence of a stroke mimic. Patients who had a diagnosis of cerebral ischemia confirmed by neurologists experienced a significantly higher subsequent rate of stroke compared to patients with alternate diagnoses. Physicians should have a high index of suspicion of stroke and TIA in patients with advanced age, smoking history, language disturbance, or infarcts on CT. Physicians should think carefully before pursuing stroke investigations or initiating therapies in patients deemed at minimal risk of cerebral ischemia, including those presenting with vertigo in the absence of 
other findings of posterior circulation cerebral ischemia, confusion, syncope, or bilateral symptoms.

Supplementary Information The online version contains supplementary material available at https://doi.org/10.1007/s43678-021-00181-0.

Acknowledgements The authors would like to acknowledge peerreviewed funding from the Canadian Institutes of Health Research. Dr. Jacques Lee is supported by the Schwartz/Reisman Emergency Medicine Institute Inaugural Research Chair in Geriatric Emergency Medicine. Dr. Jeffrey Perry is supported by the Ontario Heart and Stroke Foundation with a Mid-Career Award. The authors would also like to thank the hundreds of physicians who completed our data collection forms and all the emergency department nurses and clerks at the 13 study sites for their cooperation with the study.

Funding Dr. Perry is supported by a mid-career award from the Heart and Stroke Foundation of Ontario. Dr. Lee is supported by the Schwartz/Reisman Emergency Medicine Institute Inaugural Research Chair in Geriatric Emergency Medicine. No other relationships or activities that could appear to have influenced the submitted work.

\section{Declarations}

Conflict of interest The authors declare that they have no conflict of interest.

Guarantor information The guarantor, Jeffrey Perry accepts full responsibility for the work and/or the conduct of the study, had access to the data, and controlled the decision to publish. Dr. Perry affirms that this manuscript is an honest, accurate and transparent account of the study being reported and no important aspects of the study have been omitted, and that any discrepancies from the study as planned have been explained. The corresponding author attests that all listed authors meet authorship criteria and that no other meeting the criteria have been omitted.

\section{References}

1. Simpson KN, Simpson AN, Hill MD, Palesh YY, Jauch EC, Khatri P, Kleindorfer D, Foster LD, Mauldin PD, Broderick JP, and for the IMS III Investigators. Abstract 185: residual stroke morbidity and post-acute care cost: the IMS III Economic data cohort. Stroke. 2016;47:A185. https://doi.org/10.1161/str.47. suppl_1.185Stroke.

2. Marini C, De SF, Sacco S, et al. Contribution of atrial fibrillation to incidence and outcome of ischemic stroke: results from a population-based study. Stroke. 2005;36:1115-9.

3. Johnston SC, Gress DR, Browner WS, Sidney S. Short-term prognosis after emergency department diagnosis of TIA. JAMA. 2000;284:2901-6.

4. Canadian Institute for Health Information. Patient Cost Estimator. 2016. Ottawa, Canadian Institute for Health Information. Ref Type: Online Source.

5. Gladstone DJ, Kapral MK, Fang J, Laupacis A, Tu JV. Management and outcomes of transient ischemic attacks in Ontario. Can Med Assoc J. 2004;170:1099-104.

6. Wu CM, McLaughlin K, Lorenzetti DL, Hill MD, Manns BJ, Ghali WA. Early risk of stroke after transient ischemic attack: a systematic review and meta-analysis. Arch Intern Med. 2007;167:2417-22.
7. Amarenco P, Lavallee PC, Labreuche J, et al. One-year risk of stroke after transient ischemic attack or minor stroke. N Engl J Med. 2016;374:1533-42.

8. Buisman LR, Tan SS, Nederkoorn PJ, Koudstaal PJ, Redekop WK. Hospital costs of ischemic stroke and TIA in the Netherlands. Neurology. 2015;84:2208-15.

9. Wiest PW, Locken JA, Heintz PH, Mettler FA Jr. CT scanning: a major source of radiation exposure. Semin Ultrasound CT MR. 2002;23:402-10.

10. Shoamanesh A, Charidimou A, Sharma M, Hart RG. Should patients with ischemic stroke or transient ischemic attack with atrial fibrillation and microbleeds be anticoagulated? Stroke. 2017;48:3408-12.

11. Zhu W, He W, Guo L, Wang X, Hong K. The HAS-BLED score for predicting major bleeding risk in anticoagulated patients with atrial fibrillation: a systematic review and meta-analysis. Clin Cardiol. 2015;38:555-61.

12. Lee W, Frayne J. Transient ischaemic attack clinic: an evaluation of diagnoses and clinical decision making. J Clin Neurosci. 2015;22:645-8.

13. Boulanger JM, Lindsay MP, Gubitz G, et al. Canadian stroke best practice recommendations for acute stroke management: prehospital, emergency department, and acute inpatient stroke care, 6th edition, update 208. Int J Stroke. 2018;13:949-84.

14. Moeller JJ, Kurniawan J, Gubitz GJ, Ross JA, Bhan V. Diagnostic accuracy of neurological problems in the emergency department. Can J Neurol Sci. 2008;35:335-41.

15. Karlinski M, Gluszkiewicz M, Czlonkowska A. The accuracy of prehospital diagnosis of acute cerebrovascular accidents: an observational study. Arch Med Sci. 2015;11:530-5.

16. Kothari RU, Brott T, Broderick JP, Hamilton CA. Emergency physicians. Accuracy in the diagnosis of stroke. Stroke. 1995;26:2238-41.

17. Morgenstern LB, Lisabeth LD, Mecozzi AC, et al. A population-based study of acute stroke and TIA diagnosis. Neurology. 2004;62:895-900.

18. Perry JJ, Sharma M, Sivilotti ML, et al. A prospective cohort study of patients with transient ischemic attack to identify highrisk clinical characteristics. Stroke. 2014;45:92-100.

19. Perry JJ, Sivilotti MLA, Emond M, et al. Prospective validation of Canadian TIA Score and comparison with ABCD2 and $\mathrm{ABCD} 2 \mathrm{i}$ for subsequent stroke risk after transient ischaemic attack: multicentre prospective cohort study. BMJ. 2021;372:n49.

20. R Core Team. R: a language and environment for statistical computing. 2019. Vienna, Austria, R Foundation for Statistical Computing. Ref Type: Online Source.

21. Harrell F. Regression modeling strategies with applications to linear models, logistic and ordinal regression and survival analysis. 2nd ed. New York: Springer-Verlag; 2016.

22. Gibson LM, Whiteley W. The differential diagnosis of suspected stroke: a systematic review. J R Coll Physicians Edinb. 2013;43:114-8.

23. Merino JG, Luby M, Benson RT, et al. Predictors of acute stroke mimics in 8187 patients referred to a stroke service. J Stroke Cerebrovasc Dis. 2013;22:e397-403.

24. Tarnutzer AA, Lee SH, Robinson KA, Wang Z, Edlow JA, Newman-Toker DE. ED misdiagnosis of cerebrovascular events in the era of modern neuroimaging: a meta-analysis. Neurology. 2017;88:1468-77.

25. Yu AYX, Hill MD, Asdaghi N, et al. Sex differences in diagnosis and diagnostic revision of suspected minor cerebral ischemic events. Neurology. 2021;96:e732-9.

26. Gargano JW, Wehner S, Reeves MJ. Do presenting symptoms explain sex differences in emergency department delays among patients with acute stroke? Stroke. 2009;40:1114-20. 
27. Stuart-Shor EM, Wellenius GA, DelloIacono DM, Mittleman MA. Gender differences in presenting and prodromal stroke symptoms. Stroke. 2009;40:1121-6.

28. Merikangas KR. Contributions of epidemiology to our understanding of migraine. Headache. 2013;53:230-46.

29. MacClellan LR, Giles W, Cole J, et al. Probable migraine with visual aura and risk of ischemic stroke: the stroke prevention in young women study. Stroke. 2007;38:2438-45.

30. Schurks M, Rist PM, Bigal ME, Buring JE, Lipton RB, Kurth T. Migraine and cardiovascular disease: systematic review and meta-analysis. BMJ. 2009;339:b3914.

31. Spector JT, Kahn SR, Jones MR, Jayakumar M, Dalal D, Nazarian $\mathrm{S}$. Migraine headache and ischemic stroke risk: an updated metaanalysis. Am J Med. 2010;123:612-24.
32. Yu AYX, Penn AM, Lesperance ML, et al. Sex differences in presentation and outcome after an acute transient or minor neurologic event. JAMA Neurol. 2019;76:962.

33. Newman-Toker DE, Hsieh YH, Camargo CA Jr, Pelletier AJ, Butchy GT, Edlow JA. Spectrum of dizziness visits to US emergency departments: cross-sectional analysis from a nationally representative sample. Mayo Clin Proc. 2008;83:765-75.

34. Nouh A, Remke J, Ruland S. Ischemic posterior circulation stroke: a review of anatomy, clinical presentations, diagnosis, and current management. Front Neurol. 2014;5:30.

35. Coutts SB, Moreau F, Asdaghi N, et al. Rate and prognosis of brain ischemia in patients with lower-risk transient or persistent minor neurologic events. JAMA Neurol. 2019;76:1439.

\section{Authors and Affiliations}

Miguel A. Cortel-LeBlanc ${ }^{1} \cdot$ Mukul Sharma $^{14} \cdot$ Achelle Cortel-LeBlanc $^{3} \cdot$ Marco L. A. Sivilotti ${ }^{4}$ Marcel Émond ${ }^{5,6}$. Ian G. Stiell 1,2 . Grant Stotts ${ }^{7}$. Jacques Lee ${ }^{8,9} \cdot$ Andrew Worster $^{10}$. Judy Morris ${ }^{11} \cdot$ Ka Wai Cheung $^{12}$.

Albert Y. Jin ${ }^{13}$. Wieslaw J. Oczkowski ${ }^{14}$. Demetrios J. Sahlas ${ }^{14} \cdot$ Heather E. Murray ${ }^{4}$. Ariane Mackey ${ }^{5,15}$. Steve Verreault ${ }^{5,15} \cdot$ Marie-Christine Camden $^{5,15} \cdot$ Samuel Yip $^{16} \cdot$ Philip Teal $^{16} \cdot$ David J. Gladstone $^{17}$. Mark I. Boulos ${ }^{17}$. Nicolas Chagnon ${ }^{18}$. Elizabeth Shouldice ${ }^{3}$. Clare Atzema ${ }^{9}$. Tarik Slaoui ${ }^{11}$ - Jeanne Teitelbaum ${ }^{11}$. Kasim E. Abdulaziz ${ }^{2} \cdot$ George A. Wells $^{2} \cdot$ Monica Taljaard $^{2} \cdot$ Jeffrey J. Perry ${ }^{1,2} \mathbb{D}$

1 Department of Emergency Medicine, University of Ottawa, Ottawa, ON, Canada

2 Clinical Epidemiology Unit, F647, The Ottawa Hospital, Ottawa Hospital Research Institute, 1053 Carling Avenue, Ottawa, ON K1Y 4E9, Canada

3 Queensway Carleton Hospital and University of Ottawa, Ottawa, ON, Canada

4 Department of Emergency Medicine, Queen's University, Kingston, ON, Canada

5 CHU de Québec, Hôpital de l'Enfant-Jésus, Québec City, QC, Canada

6 Division of Emergency Medicine, Université Laval, Québec City, QC, Canada

7 Division of Neurology, Department of Medicine, University of Ottawa, Ottawa, ON, Canada

8 Schwartz/Reisman Emergency Medicine Institute, Mount Sinai Hospital, Toronto, ON, Canada

9 Department of Emergency Medicine, Sunnybrook Health Sciences Centre, Toronto, ON, Canada
10 Division of Emergency Medicine, McMaster University, Hamilton, ON, Canada

11 Hôpital du Sacré-Cœur de Montréal, Université de Montréal, Montréal, QC, Canada

12 University of British Columbia, Vancouver, BC, Canada

13 Division of Neurology, Queen's University, Kingston, ON, Canada

14 Division of Neurology, McMaster University, Hamilton, ON, Canada

15 Division of Neurology, Laval University, Quebec City, QC, Canada

16 Division of Neurology, University of British Columbia, Vancouver, BC, Canada

17 Sunnybrook Research Institute and Division of Neurology, Department of Medicine, Sunnybrook Health Sciences Centre and University of Toronto, Toronto, ON, Canada

18 Department of Emergency Medicine, Montfort Hospital and University of Ottawa, Ottawa, ON, Canada 